\title{
Conceptual Framework for SDSS Development with an Application in the Retail Industry
}

\author{
Gautier Daras · Bruno Agard · Bernard Penz
}

Received: 9 September 2017 / Accepted: 2 May 2018/Published online: 20 June 2018

(C) Springer Fachmedien Wiesbaden GmbH, part of Springer Nature 2018

\begin{abstract}
Spatial information is becoming crucial for strategic decision making, but accessing and understanding this information is not easy. Dedicated tools can support the decision process in many ways, such as visualization interfaces or data analyses. Numerous Decision Support System (DSS) development methodologies exist along with dedicated Spatial Decision Support System (SDSS). Unfortunately, for multiple reasons, these tools and methodologies are not easily adaptable for the development of another SDSS. This paper proposes a framework for the development of a flexible SDSS that is built on open source software, allowing for low cost implementation. To support the efficiency of our approach, the design of a specific SDSS that is currently in use will be presented. This SDSS was developed for a company that distributes products through various retail networks. The multiple capabilities of the resulting SDSS will be revealed through an explanation of the different development steps. The complete framework is applied to a real data set that will be detailed in a demonstration.
\end{abstract}

Keywords SDSS · Framework · Development · Case study $\cdot$ Retail

Accepted after one revision by Prof. Dr. Kliewer.

G. Daras $(\bowtie) \cdot$ B. Agard

FORAC, CIRRELT, Département de Mathématiques et Génie Industriel, École Polytechnique de Montréal, Montreal, QC H3T 1J4, Canada

e-mail: gautier.daras@polymtl.ca

B. Agard

e-mail: bruno.agard@polymtl.ca

G. Daras $\cdot$ B. Penz

Univ. Grenoble Alpes, CNRS, Grenoble INP, G-SCOP,

38000 Grenoble, France

e-mail: bernard.penz@grenoble-inp.fr

\section{Introduction}

For decades, public and private organizations from various domains have accumulated large amounts of information about their day to day activities. In many cases, collected data includes spatial references that may describe where an activity occurred. Nonetheless, the storage, management and use of spatialized data is still a challenge, and Erskine et al. (2013) and MacEachren and Kraak (2001) have highlighted the need to develop those topics in order to simplify strategic and organizational decision making.

Researchers have focused on many of these topics, and as a result, more and more tools are available to exploit spatial information to assist decision making. Tools developed to achieve this goal are frequently called Spatial Decision Support System (SDSS) as introduced by Armstrong et al. (1990) and Densham (1991) in the early 1990s. Then, Crossland et al. (1995) state that SDSS allows for improvement in decision making performance. While many SDSS development projects were made, there are still challenges noted in the literature. Hernandez (2007) claimed that a part of the challenge is to provide functionalities that meet the needs of decision makers and that facilitate adequate visualization of available data. Sugumaran (2007) highlighted the need to build an intelligent SDSS that uses available technologies and facilitates the interoperability of spatial data and systems. For Keenan (2004), an appropriate synthesis of modeling techniques, interfaces and database approaches needs to be developed.

Knowledge about spatial environments may be a key element in improving understanding and performance in many fields, in particular for the retail sector (Cliquet et al. 2006; Dubelaar et al. 2002) which is facing many challenges (Reinartz et al. 2011). The growing interest in techniques for the analysis and understanding of spatialized 
data follows growing competitiveness (Thompson and Walker 2005). More recently, Bradlow et al. (2017) insist on the growth of the role of data analysis in retail. Keenan (2006) identified that top managers need spatial information analysis and Mendes and Themido (2004) outlined that top managers are able to express their needs. Unfortunately, most managers do not have the time and interest to learn how to use complex spatial data analysis systems or to develop their own mathematical models. In many situations, a lack of commercial solutions leads to the development of dedicated SDSS solutions [as in Wanderer and Herle (2015), Zhang et al. (2010) for example]. Unfortunately, the developments recommended in those types of research are difficult or impossible to adapt to different case studies. Table 1 in Sect. 2.3 exposes the main reasons for inadaptability that are encountered.

The contribution of this research is not in the development of a new theory, but in the proposition of a new conceptual framework that is easily adaptable to the development of many types of SDSS. The proposed approach allows for an integration of various existing methods and tools. For better clarity, demonstrations will be based on a real case study. While SDSS might focus on many research areas related to retail, we will consider the development of a SDSS dedicated to the evaluation of the commercial network of our partner company: Maibec (2016). Maibec is a company specialized in siding systems, which distributes its products through its partner's points of sale. The company wanted to expand its distributor network, and wanted to take advantage of the data available to them. Their partnership lies in providing their data, business expertise and ongoing feedback on the proposed Decision Support System.

The next Sect. 2 presents relevant research in the domain. It focuses on the design principles and development architecture requirements as well as various methods. Section 3 expands upon the contributions of the paper. In Sect. 3.1, the context of the case study is introduced. Section 3.2 explains the approach proposed in this paper, the architecture and selected tools will be highlighted. Then, in Sect. 3.3, a step by step explanation, based on the case study, enables the proposed approach to be clearly illustrated. In this section, the needs of the case study are formalized. This shows how to meet those needs in the proposed framework along with the results of this implementation. Section 3.4 aims to demonstrate the adaptability of the SDSS by transferring previously developed applications to another geographic area. The final Sect. 4 concludes the paper and proposes future research directions.

All steps in this contribution have been validated in the case study with real data from the researchers' partner along with various public and private data. For reasons of confidentiality, all financial parameters included here have been transformed or masked.

\section{Literature Review}

Technologies linked to SDSS development evolve rapidly and it is possible to identify several papers that focus on design principles and development architecture requirements (Sect. 2.1). Some design and development methodologies from related fields including Decision Support System (DSS) and Knowledge Discovery from Databases (KDD) are also presented (Sect. 2.2).

\subsection{Design Principles and Requirements of Development Architecture}

According to many researchers (Densham 1991; Keenan 2004; Khan and Khan 2011), SDSS consists in three main fields: (1) data integration and manipulation, (2) analysis, and (3) visualization of the results.

(1) As mentioned by Otto (2011), data quality is critical for enterprises if they want to meet their business requirements. However, the initial data quality is not sufficient with regards to spatial data integration; Densham (1991) highlighted the need to provide mechanisms for the input and integration of spatial data in a flexible manner, in a way that facilitates further analysis. With regards to those assertions and to the spatial aspect of data treatment, Evans and Sabel (2012) pointed out the efficiency of the PostgreSQL solution (with the PostGIS extension) that allows spatial queries on the data to be made.

(2) On a general design aspect on spatial data analysis, SDSS are supposed to include analytical techniques that are unique to both spatial and geographical analysis (including statistics) (Densham 1991) and they must be able to adapt to the needs of the users as they evolve. On the closely related area of reusability, more recently, Wanderer and Herle (2015) advanced that it must be profitable to allow different models of analysis to be plugged into the same framework. In a more technical aspect, Zhang et al. (2010) insisted on examining the performance aspect if models are applied on the user's computer. Also in relation to the performance, Vatsavai et al. (2006) pointed out that, in a classic fat server-light clients approach, the server could be overburdened by data access and spatial analysis, while in the thick client, light server approach, the same limitations could occur on the client side. 
Table 1 Difficulties identified in transfering other research to various contexts

\begin{tabular}{|c|c|c|c|}
\hline References & Methodology & $\begin{array}{l}\text { Case Main focus } \\
\text { study }\end{array}$ & $\begin{array}{l}\text { Difficulties identified to transfer to various } \\
\text { contexts }\end{array}$ \\
\hline
\end{tabular}

Agrawal and Gupta $\quad x$

(2014)

Bogorny et al. (2005)

$\mathrm{x}$

Chen et al. (2008)

Cios et al. (2007),

Fayyad et al. (1996)

Evans and Sabel

(2012)

$\mathrm{x} \quad$ Interfaces interaction visualization techniques

KDD process

Comparison of open source web- There is no possibility to implement based GIS

Data preparation$$
\text { cess }
$$

Open source SDSS for health and environment

Granell et al. (2010)

Jin (2011)

$\mathrm{X}$

$\mathrm{x}$

Knezic and Mladineo $\mathrm{x}$ (2006)

Loucks (1995) x

Moreno-Sanchez

et al. (2007)

Rinner et al. (2008)

Web framework for deliberation

Roig-Tierno et al. $\quad \mathrm{x}$ (2013)

Sikder (2009)

Sprague (1980) X

Wanderer and Herle (2015)

Zhang et al. (2010)

Zhu et al. (1998)
Design and implementation of web-based platform

$\mathrm{x}$

$\mathrm{x}$ in spatial decision making

$\begin{array}{lll}\mathrm{x} & \mathrm{x} & \begin{array}{l}\text { Design and implementation of } \\ \text { web-based platform }\end{array} \\ \mathrm{x} & \mathrm{x} & \begin{array}{l}\text { Reusable geospatial services } \\ \text { Web DSS for e-retail industry }\end{array} \\ \mathrm{x} & & \end{array}$

$\mathrm{x}$

$\mathrm{x}$

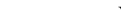

Retail site location decision process

$\mathrm{X}$

SDSS in environmental assessment

DSS development framework

x $\quad$ Multi criteria SDSS

Web-based SDSS framework

Knowledge-based approach for SDSS development advanced model analysis

There is no open source tool proposed

The user interface is not taken into account

There is no global architecture proposed

There is no open source tool proposed

There is only methodology

There is no tool proposed

There is no user interface

There is no possibility to implement advanced model analysis

There is no tool proposed for development

There is no spatial data treatment tool for advanced spatial data manipulation

There is no open source tool proposed

Not on dedicated SDSS development

Tools used are not specified

There is no open source tool proposed

There is no open source tool proposed

There is no open source tool proposed

There is no spatial data treatment tool for advanced spatial data manipulation

There is no possibility to implement advanced model analysis

There is no spatial data treatment tool for advanced spatial data manipulation

There is no possibility to implement

The proposed process is dedicated to making a decision about a retail location

There is no tool proposed for development

Tools used are not specified

There is no open source tool proposed

There is no open source tool proposed

There is no spatial data treatment tool for advanced spatial data manipulation

There is no open source tool proposed

There is no spatial data treatment tool for advanced spatial data manipulation

There is no open source tool proposed advanced model analysis
(3) Regarding the visualization part, Lloyd (1997) advanced that SDSS often use Geo-visualization techniques because humans learn more easily and efficiently when the support is visual rather than when it is textual or numerical. Also regarding visualiza tion Khan and Khan (2011) said that users 
are interested in simplified information and presented various visualization techniques that improve upon an understanding of the data. In a closely related field, Evans and Sabel (2012) presented techniques that could be included to improve usability, such as the addition or removal of information on a map, or a focus on specific aspects. Also, to improve understanding and exploration, Knezic and Mladineo (2006) presented a multi-level approach that allows for a change in spatial granularity. As all of those interactions are not easy to develop from the very beginning, the architecture must include libraries that allow for those kinds of interactions, such as Openlayers, which is presented as an efficient solution by Agrawal and Gupta (2014).

Two other points that are not specific to SDSS are accessibility and cost. On accessibility, Granell et al. (2010) argued that web-based tools, including web-based SDSS, do not need any software to be installed so that they can be used by anybody, anywhere. Rinner et al. (2008) added that there is a growing availability of free web services, which do not need knowledge of GIS in order to be used [such as Google Maps (Google 2016)].

Concerning costs, many researchers state thisit is one of the advantages of open source technologies (Rey 2009; Moreno-Sanchez et al. 2007; Evans and Sabel 2012). Moreover, large open source communities are devoted to helping and sharing ideas (Evans and Sabel 2012).

There is little research linked to the SDSS development process and methodologies. However, research has focused on similar domains such as DSS (Decision Support System) or KDD (Knowledge Discovery in Databases) applications. In the next section, the most accepted methodologies will be presented. Identified weaknesses when dealing with custom SDSS development will be indicated.

\subsection{Design and Development Methodologies}

Many papers (Sprague 1980; Cios et al. 2007; Fayyad et al. 1996 for example) have identified the process of extracting information from data in decision systems. This development process is composed of six main steps. Step 1 understanding the problem domain: defining the problem, determining the project goals, identifying key people and domain-specific terminology. Step 2 - data collection, selection and cleaning. Step 3 - data preparation, which consists of numerous tasks that allow the data to be transformed from its original format to a dataset that is adapted for a specific intented purpose (such as applying data mining algorithms). Step 4 - selection of appropriate
Data mining methods. Step 5 - evaluation of the discovered knowledge. Step 6 - use of the discovered knowledge. This process is not linear and multiple steps back are usually necessary.

A common point found in the literature is that domain knowledge is essential to DSS development. It allows for better comprehension of the problem and increases the quality of the developed system (Adejuwon and Mosavi 2010; Hess et al. 2004).

Cios et al. (2007) and Bogorny et al. (2005) insisted that data preparation is very time consuming (generally more than fifty percent of the total project duration). Moreover, other problems will appear when dealing with spatial data preparation (Mennis and Guo 2009; Flowerdrew 1991), such as measurement uncertainty, biased sampling, varying area unit, etc.

Regarding DSS development, Sprague (1980) said that during the development process, the system definitions and objectives change frequently, and this has to be taken into account by the user and the developer. The development framework must permit quick and easy changes. Loucks (1995) added that during the whole development process, people are involved learn more about what they can have, and their needs may evolve as they become better informed.

\subsection{Synthesis}

According to what has been found in recent scholarly publications, many methodologies in close fields have been proposed and various SDSS have been developed. Those contributions may be separated into two categories: generic methodologies and case-based specific applications.

Generic methodologies mainly focus on DSS development and KDD process but do not include spatial specificity, such as spatial data preparation and analyses that have been presented in some research (Bogorny et al. 2005; Flowerdrew 1991). As those methodologies do not include this saptial aspect, it is difficult to apply them to the development of a SDSS.

Case study papers present SDSS developed specifically for a situation, but proposed functionalities may not correspond to someone else's needs. Even if the need is the same, adapting those SDSS may be difficult or impossible because of the technology costs or missing information about involved technologies. Most of the time, authors explain the functionalities of the tools developed but not how they were developed, what kind of database, what kind of objects, or which software were used (see Table 1). In other cases, those elements are clearly explained but the functionalities are too specific and cannot be adapted easily in another situation (see Table 1). 
Benoit and Clarke (1997) said that simple models are unlikely to succeed in many applied market situations and that customized solutions will lead to a better capture of complex situations. While many methodologies look like the generic one presented by Cios et al. (2007), they often aim at one model project. Yet, many companies might need several decision tools. The data preparation phase is tedious and if not made in an adaptable way, it might have to be re-done for every situation. Furthermore, this adaptability is even more critical considering the evolving nature of custom SDSS as decision makers clarify their needs.

SDSS tools and methodologies are currently developed in many application domains such as environment (Ghaemi et al. 2009; Granell et al. 2010; Wanderer and Herle 2015), healthcare (Moreno-Sanchez et al. 2007), agriculture (Sikder 2009) and others (Roig-Tierno et al. 2013; Jin 2011; Rinner et al. 2008) and may be useful in many other research andprofessional domains.

Table 1 makes a synthesis of the difficulties encountered when trying to adapt other SDSS to our case study. The difficulties encountered in those papers relative to the flexibility are identified. The identified papers has been gathered to cover various fields and to highlight the current gaps in the literature. The focus is not being exhaustive, but rather to highlight the need for a flexible tool that offers easy adaptability to various contexts.

Considering these elements, the focus of this paper is to propose a conceptual framework, based on a SDSS architecture and a development approach that could be adapted to various distinct applications. Those may serve as a foundation for the development of any SDSS application. The proposed architecture is based only on open source software to allow for implementation with no setup costs.

The SDSS was effectively developed, and is currently in use, by the partner company.

\section{Presentation of the Framework Through a Case Study}

The dedicated SDSS and how it was implemented is presented in this section. For better clarity, the following demonstrations will be based on a real case study. This section details the case study, the architecture of the SDSS and its main functionalities, and shows its adaptability. When necessary, we explain why the proposed solution is flexible and can be used in various contexts.

\subsection{The Needs of a Retail Company}

Our industrial partner is a Canadian leader in lodge building material, based in Quebec (Canada), with a strong presence in North America, mostly on the East Coast. The partner manufactures different products and distributes them through about 700 dealers that are present in about 50 commercial networks. Considering growing competition, a consolidation and better understanding of the market and commercial performances is needed.

The first need is therefore to assess the performance of the current commercial network, and then to evaluate the impacts of any changes in this network. Many questions have arisen immediately that concern the location of the dealers on the territory, as well as individual and global performances.

Furthermore, individual and global performance depends on the context. The relevant local environmental context of each retailer needs to be extracted, in parallel to the retailer's historic sales, before any evaluation is done. The SDSS presented here will allow for all of that information to be extracted locally for each dealer and globally for the network.

\subsection{SDSS Applications, Development Process, and Tools Required}

Our proposed approach of SDSS development process (composed of six main steps: A-F) is represented in Fig. 1. This figure presents a simplified view of the workflow, that does not aspire to be a theoretical methodology for SDSS development. This workflow will on one hand structure the

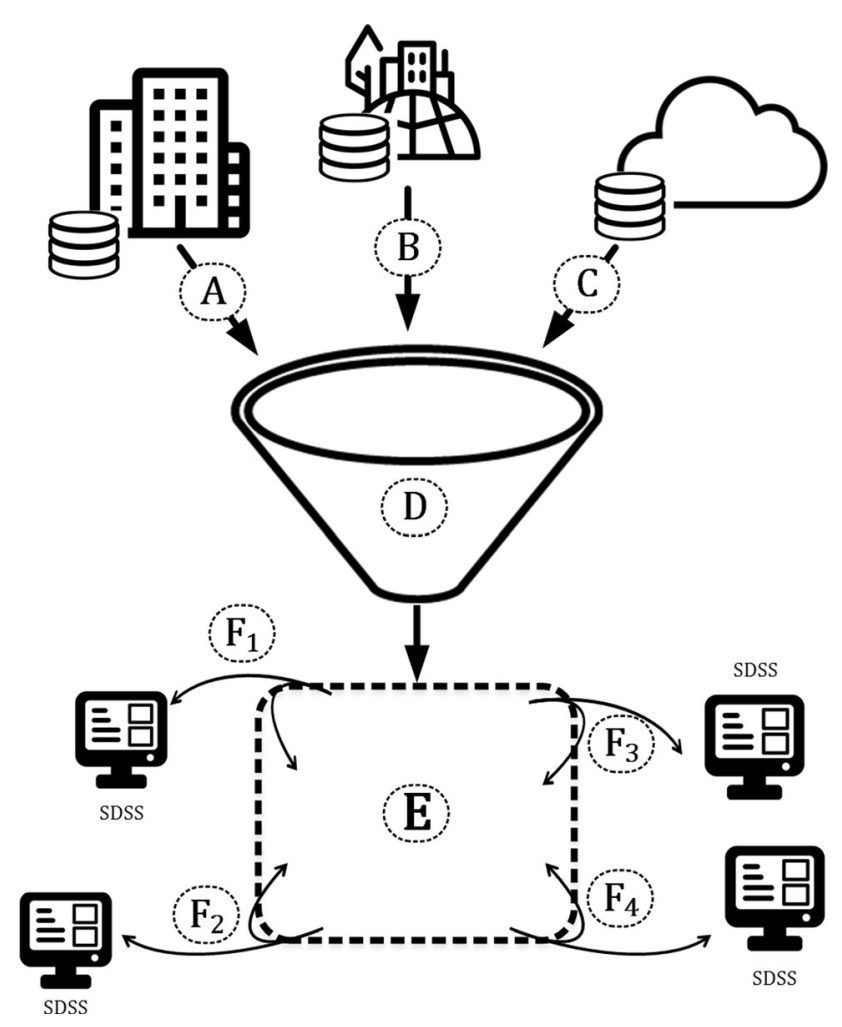

Fig. 1 SDSS development workflow 
selection of the necessary tools to develop the SDSS. On the other hand, it supports the development timeline of our case study in Sect. 3.3.

Steps A, B and C consist of data collection. Many sources may be concerned as soon as those sources provide useful information for the case study. In each case study, the collected data vary and this step needs to be carefully observed. This is a critical aspect and bad data collection will simply lead to no answer, or worse, to wrong answers in the final steps.

Currently, many datasets about socio-demographic data, statistics, roads, circulation, and so on exist and can be downloaded from different places. It is important to carefully search the relevant sets of data and to be sure that the data is clean, structured and understood. Synthetic data sets are usually poor and may not be useful. Disaggregated data sets are much richer but it may take time to prepare them for analysis.

For the case study presented here, among the different datasets used, the following are the most important:

$A$ - the company provided raw data on a retailer's sales per month. The initial dataset concerned the province of Quebec (Canada). For each retailer, the following data was available: name, address, partner representative, retail network, and the sales of each month.

$B-$ as the sales are influenced by socio-demographic characteristics, specific points of interest and construction activities, the following data were included:

- socio-demographic data from the national census bureau (Statistics-Canada 2016),

- locations of golf courses (Golf-clubs 2016), Skiresort (2016) and large lakes (Statistics-Canada 2016) (as our partner thought that those might influence sales performances),

- statistics on construction: building permits (Statistics-Canada 2016) and housing starts ${ }^{1}$ (CMHC 2016).

Some of these datasets had to be purchased from Canadian Mortgage and Household Corporation (CMHC) and Statistics Canada.

$C$ - description of the geographical territory.

The province of Quebec is subdivided into areas of various sizes. Different levels of aggregation are available; here the "Census Subdivision"? (CSD) was selected. This is a level that satisfies both the level and accuracy that is expected for the case study and conforms to the datasets available in A and B.

Depending on the project's needs, other area sizes might

\footnotetext{
${ }^{1}$ Housing starts is an economic indicator that reflects the number of privately owned new houses on which construction has been started in a given period.
}

be chosen, such as Province or Census Division (CD). As the future steps propose developing a generic data treatment and tools, the adaptation to other areas will be easy. All of these datasets cannot be imported as they are in the SDSS database. Data treatments and integrations presented in part $\mathrm{D}$ focus on this.

$D$ - data treatment and integration.

As mentioned in the literature review, data treatment and integration are tedious and complicated tasks. Also, with the goal of developing flexible SDSS, those tasks may be repeated several times and must be imported properly. Moreover, the different datasets in A, B and C may be in different formats and may contain various problems. For each dataset, a specific engine may be required; those engines have to identify errors and adapt the data format to the SDSS database in E. Among the engines used for our case study, the following are crucial:

- address Geocoding (convert an address to geographical coordinates),

- duplicate removal,

- area simplification (to improve interface responsiveness),

- XML, HTML and CSV data parsing,

- inconsistent data homogenization (punctuation, accents, etc).

This step will allow for clean datasets in the input for the development of the SDSS (step F). Moreover, even though it was not the case in our problem, it is important to ensure the homogeneity of the data, whose definitions can vary according to location, such as those related to languages, time zones and currencies.

$E$ - a global architecture based on flexible tools.

This section describes the architecture and tools recommended to develop a flexible SDSS. In order to avoid server or client overload, two distinct categories of users were considered, with different needs and permission to access the server (see Fig. 2).

The first category of users is called Thick clients. Here, we find users that will proceed to the analysis, modeling and development stages. Those users can read as well as write on the database located on the server; in addition, most analyses and computation run on their own computers. The second category is called Thin clients, they have read only access to the database. They use the tools and interfaces developed by the Thick clients. For them, the server supports the computing; it enables access to the visualization interfaces through web browsers. It allows flexible access with any thin-client device, such as small computers, tablets, and smartphones.

The global architecture, presented in Fig. 3, shows a set of efficient tools selected for the data integration and for 
Fig. 2 Schematic architecture

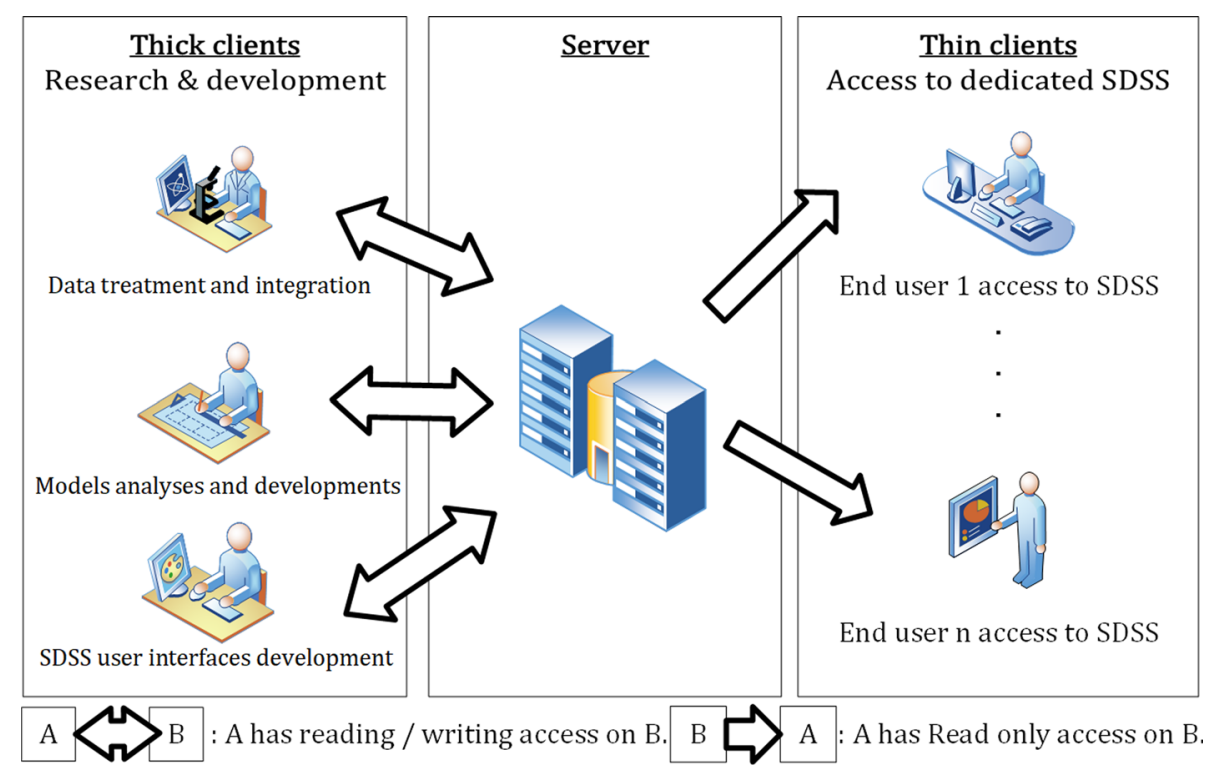

Fig. 3 SDSS architecture and development toolkit

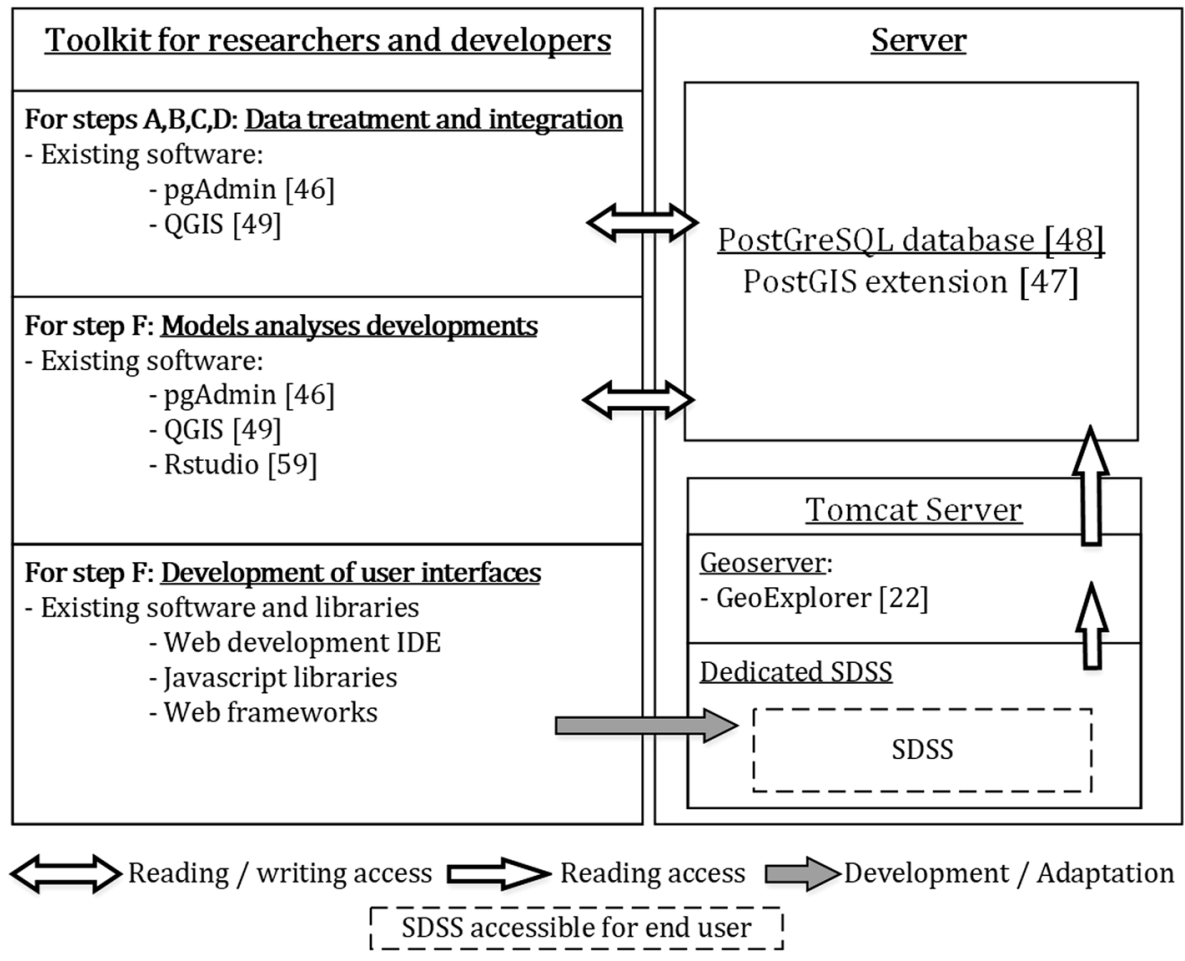

- many of those tools can be found from the BoundlessGeo (2016).

The architecture is composed of two main parts, (1) the toolkit for the researchers and developers and (2) the server.

(1) The toolkit will allow three main tasks to be performed, data treatment and integration, model analysis development, and user interface development: 
- For data treatment and integration, PgAdmin (2016) allows for the database to be administrated and for manipulations on stored data to be performed. A GIS software is included for the manipulation and the treatment of the spatial data. For these tasks, QGIS software (2016) is proposed. In addition, different generic data treatment engines are developed during the SDSS development process to handle tasks such as removing duplicates, cleaning text, geocoding addresses.

- For the model analyses and development, as previously mentioned and pointed out in the literature (Wanderer and Herle 2015), the data might be used in multiple models. PgAdmin and QGIS might be used to perform basic analysis, but are not sufficient to process advanced modeling tasks. To overcome this, the $\mathrm{R}$ programing language (2016), easily usable through the RStudio IDE (2016), is proposed. As in other steps, models developed here are generic and reusable for multiple SDSS applications.

- For the user interface development, many open source tools and libraries exist. As it is neither our focus nor our area of expertise, no specific software is mandatory in our approach. However, for information, the tools used for the development of each web interface are cited in next Sect. 3.3.3. Once again, flexibility in interface development must be thoughtful to allow the reusability and adaptability of codes for future development (as in Sect. 3.4).

(2) The server contains the database and a web server. For data storage, a lot of databases exist on the market. Few of them allow spatial data management. PostgreSQL (2016) has been selected due to the PostGIS (2016) extension that is available and and allows for spatial data to be worked with easily, and to perform spatial queries. A Tomcat web server (2016) stores a GeoServer (2016) and the SDSS Websites. GeoServer will allow easy access to spatial content through web services and/or through GeoExplorer (2016).

$F$ - development of specific functional tools.

In A, B and C, we select relevant data for the case study. $\mathrm{D}$ proposes engines to prepare the data. $\mathrm{E}$ presents a global architecture and existing software that supports the SDSS. F focuses on the development of specific tools adapted for the case study. Thick clients (researchers and developers) have to design the tools and interfaces for the Thin clients (end users). While Fig. 1 contains four specific tools, the proposed workflow does not impose any constraint on the number of developed specific tools, as can be seen in the case study presented below (which contains seven specific tools).

The framework proposed in this paper enables the tools to be adapted to any case study. The following section details the development of various functional applications, based on the case study presented in Sect. 3.1. For example, the visualizations of retailers, network identification, sales, socio-demographic parameters around each retailer, localization of the relevant points of interests, cover optimization and area segmentation are some of the proposed functionalities.

The case study validates the proposition and shows results based on real data. Once again, the framework is very flexible and the user simply needs to change the input data A, B, C and develop any functional tool to adapt to its situation. The development of functional tools is detailed in the following Sect. 3.3, and the adaptability of the whole system is demonstrated in Sect. 3.4.

\subsection{SDSS Development}

The proposed approach was tested for the development of a real application. The dedicated functional tools were developed with a step-by-step approach, each step corresponding to a given tool. The tools were considered approved when our industrial partner validated their efficiency and their usefulness for the business. Several tools are currently used by our partner.

When toolkits and servers (Figs. 1, 3) are set up, it is possible to plug in various dedicated applications. Several applications developed for the case study are presented next. For each application $F i$, the need, the methodology, and the esulting tools are detailed.

\subsubsection{F1 - Geographic Assessment of Dealers' Sales}

Need As with many other companies, an initial assessment of the dealers' sales was provided with tables and charts. One aspect that is not easily understandable through that format is the geographical environment. Geographical assessment is a valuable insight to better understand the strengths and the weaknesses of a distribution network. The need is to have the ability to visualize the data in a suitable format.

Methodology First, data acquisition (step A) and integration (step D) were processed. For step A, the input data for each retailer was available in a tabular format. Step D 
consists of converting the addresses to corresponding geographical coordinates. For that conversion (called geocoding), a Google tool is available (2016). Google service parameters have to respect some rules, such as the address element (number, street, postal code, city), which is specifically formatted. Then a request is sent and the answer is formatted according to our system: geographical coordinates are extracted, and translated in the right projection in the database.

The model developed for this first tool consists of basic computing realized through a simple SQL request. An example is presented here that corresponds to the computation of yearly sales.

Monthly sales are saved in dedicated columns (see Table A in Fig. 4). A yearly sales column is created and computed (see Fig. 4b) resulting in a modified dataset (see Fig. 4c). The resulting dataset is then easily used at any moment. For example, it is possible to offer a visual representation of the performance of each retailer. Here, a colorimetric scale was chosen and it is easy to set specific bounds to identify a different level (strong, neutral, weak) as represented in Fig. 5a.

Results A first application was developed that aimed to present retailers' yearly sales on a map. To allow for a quick analysis, the sales are presented through a colorimetric scale. If needed, detailed sales are available through their original format by clicking on each desired retailer.

For this first application, GeoExplorer (2016) offers tools that allow easy layer management and styling (Fig. 5a). The main interface is presented in Fig. 5b. Figure $5 \mathrm{c}$, shows that the detailed information may be available after user interaction (a left click on the desired retailers).

This interface permits the easy visual representation of a retail network as well as a visual representation (green, white, red) of actual dealers' sales.

\subsubsection{F2 - Sales Aggregation Per Area}

Need While assessing each retailer's performance, decision makers may also need aggregated information for specific areas.

Methodology Step C consists of area file acquisition. As our partner did not had a predefined division of the territory for the aggregation, we had to select one adapted to the need. For this case study, the Census Subdivisions (CSD) were selected for multiple reasons: CSD areas cover the province of Quebec. Their size is adapted to obtain insightful data, and this fits with most other census datasets.

The CSD file limit consists of sets of polygons (defined by a list of geographical coordinates). For the Province of Quebec, the dataset is composed of 1285 census subdivisions, and for each one, there is a name, an identifier, and the associated polygon.

In order to perform a spatial data aggregation, spatial data queries must be processed.

Spatial queries (allowed by spatial database) permit, for example, to extract the number of specific elements in a designated area (for example, see the query in Fig. 6). It also allows the sum of the sales for all retailers in the area to be obtained (illustration in Fig. 7).
Fig. 4 Dedicated sales sum query

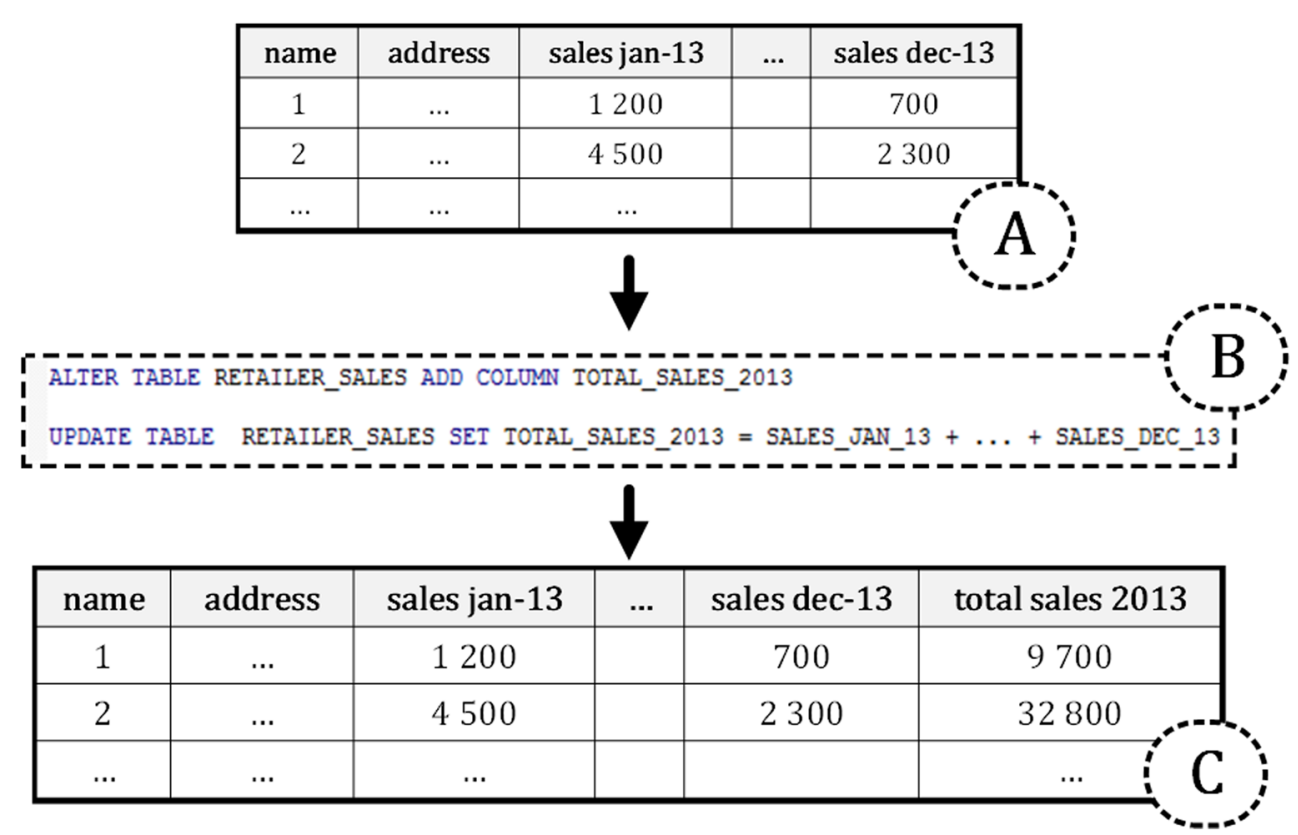




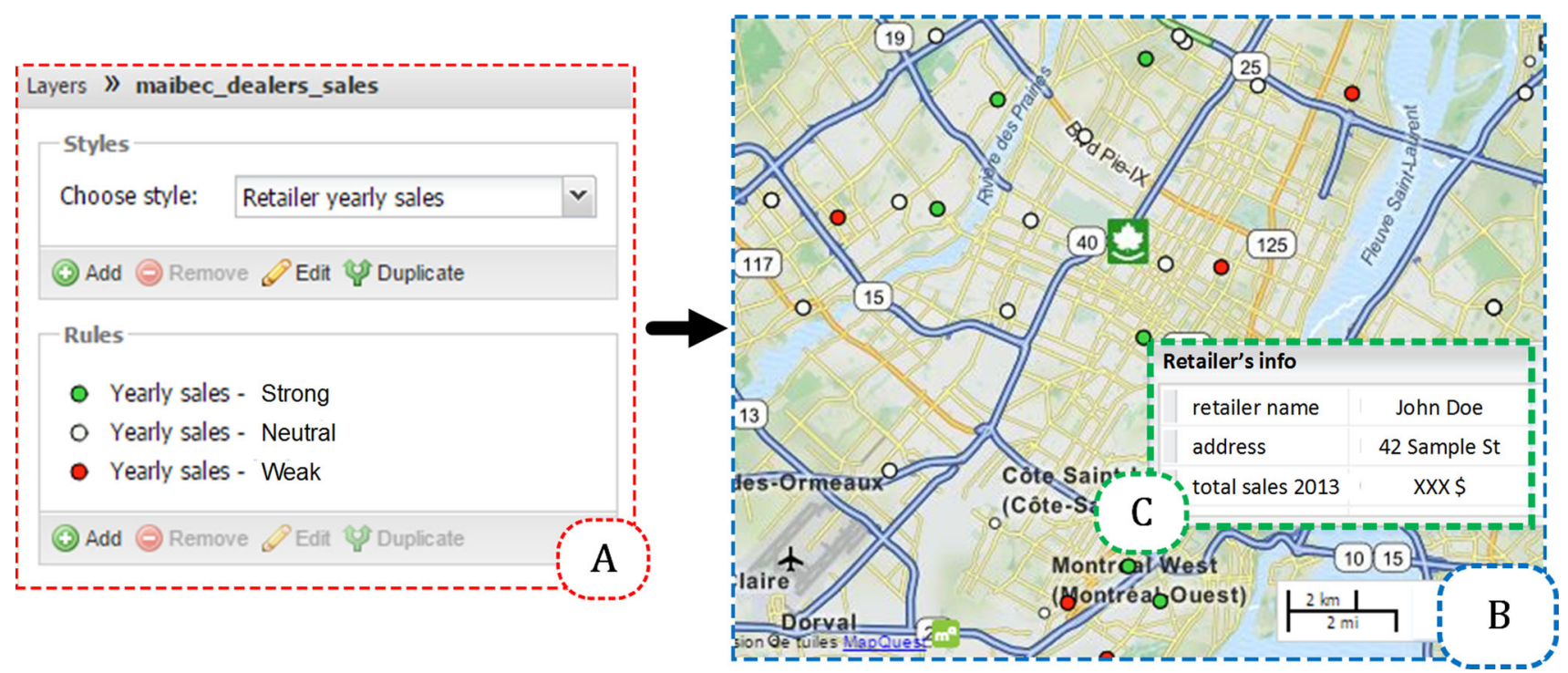

Fig. 5 First visualization interface (color figure online)

Fig. 6 Point in area cardinal query

1
2
3
4

SELECT Area.id, count(Pointofinterest) AS Cardinal FROM Area LEFT JOIN Pointofinterest ON st_contains (Area.geometry, Pointofinterest.geometry) GROUP BY Area.id
Results A second interface was developed using the GeoExplorer tool. The resulting interface is presented in Fig. 8. Areas could be colored with a colorimetric scale (the same way as presented in Fl) for any valuable information available from the retailers' dataset (monthly sales, yearly sales, etc.). The detailed information is accessible through the selection of the desired area (Fig. 8b).

This new interface allows for an aggregated evaluation to be obtained for any parameter from the retailers in each area. Then the colorimetric representation provides an easy visual representation of the results. Many area indicators could be represented (sum of the sales, average sales, etc.) that would allow different areas to be compared.

\subsubsection{F3 - Filtering Capabilities}

Need Since our partner does not sell its products directly to end customers, it makes partnerships with different retail companies. Thus, it may be pertinent to assess the retail network depending on each specified retail company. Furthermore, representatives are in charge of different retailers, and therefore it might also be of interest to get access to all retailers from a selected representative.

To meet these needs, a filtering tool was developed. It allows decision makers to focus on certain parts of the distribution network.
Methodology Data related to retailers was provided by the partner company for each retailer, the representative in charge, and the retail network company. Step A consists of formatting and importing that data.

In step F, a dedicated interface was developed. To facilitate web development, the Yeoman development stack is used (Yeoman 2016). Yeoman provides a local development environment that speeds up web interface development. Yeoman also allows for dependencies to be managed in the javascript libraries used in the project: Bootstrap (2016) for web application design, Openlayer (2016) for map visualization and interaction.

Partner networks and company representatives might change with time, thus the dedicated filters were developed generically to adapt to the retailer's current data. When the SDSS retrieves the retailer's data, it automatically extracts the existing representatives and retail networks to propose the different option in dedicated filters. This generic filtering is adaptable to any category of data (status, representative, purchasing group, etc). For numerical data, an algorithm that extracts bounding values has been developed, allowing, for example, for retailers to be filtered by turnover.

The filters on the interfaces are generated dynamically after the extraction of corresponding information. 
Fig. 7 Aggregated sales per area
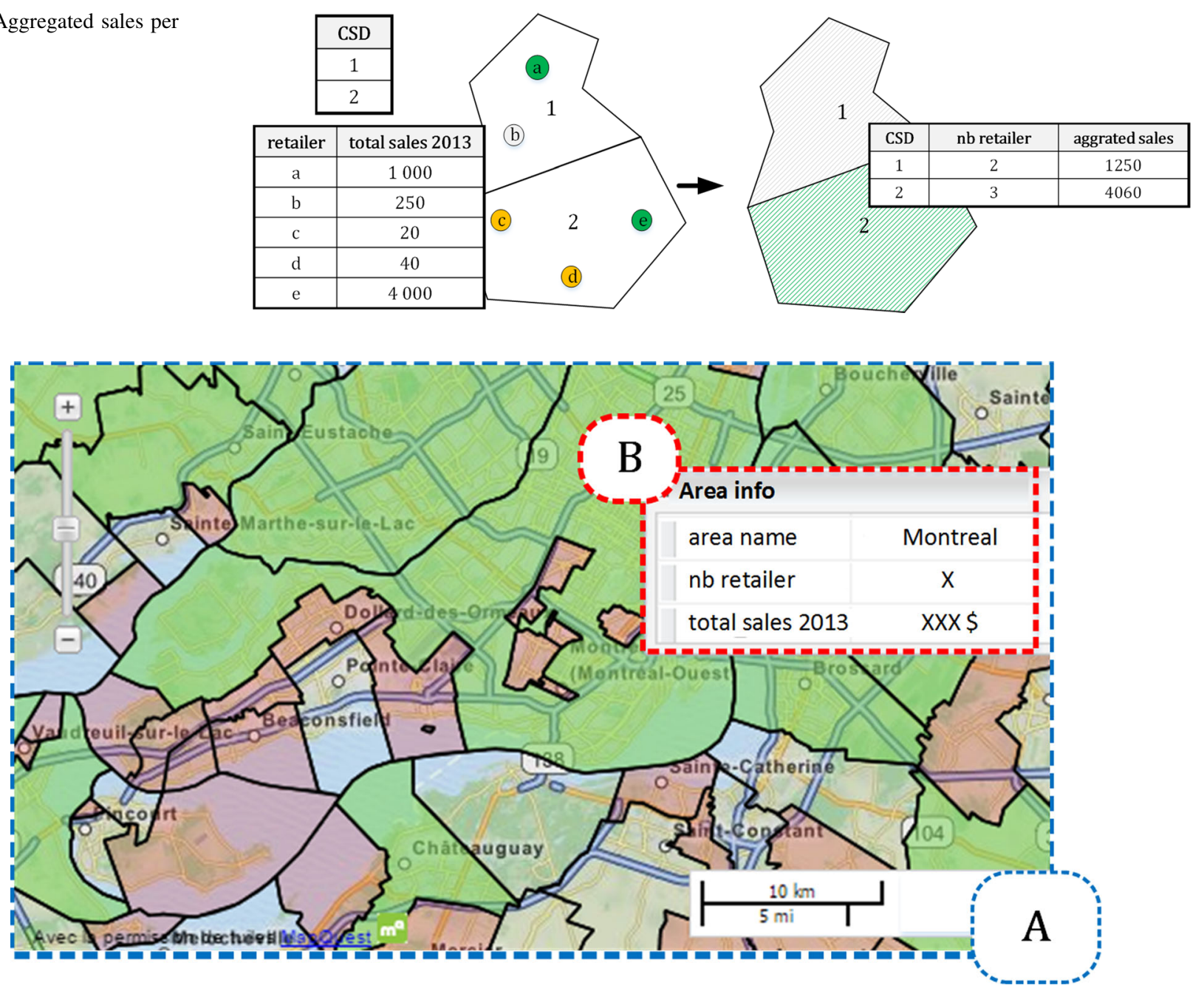

Fig. 8 Aggregated sales visualization interface

Results The resulting interface is shown in Fig. 9. While initially the filters are not set up (Fig. 9a), all the retailers appear on the map (Fig. 9b). When some filters are selected (Fig. 9c), only the corresponding retailers appear (Fig. 9d).

Filtering options allow a more precise analysis of the network. At any moment, it is possible to focus on a specific subpart of the network.

\subsubsection{F4 - Dynamical Area Polygon Styling}

Need Area limit files take a lot of memory and computing power to render on a web browser. In order to apply various styles dynamically and to get an improved user experience, CSD limits file were simplified for the visualization interfaces while original files were kept in the database to perform analysis.

Methodology The polygon simplification process is allowed by the GIS QGIS. Figure 10 illustrates the simplification process of the Montreal and Laval limits polygon, allowing for the corresponding file to go from 168 to 6 kbytes. Resulting interfaces will conserve the same information, but allow for much faster rendering and interactions. This simplification process was applied on the whole province's CSD limits file.

Results After limiting files using less memory and computing power after the simplifications, it is then possible to fluidly visualize the desired metrics through dynamic coloration of the areas. Actual drawback in the polygon simplification process is only on the graphical representation, while zooming in on the borders of some polygons we may see some zones uncovered or zones in multiple polygons. Besides, this concerns only the graphical representation of the data, and not the data by itself. Conversely, this drawback enables a huge acceleration for the visual representation. Also, it is easy to more or less simplify the polygons, depending on the performances of the tools used for visualization. 

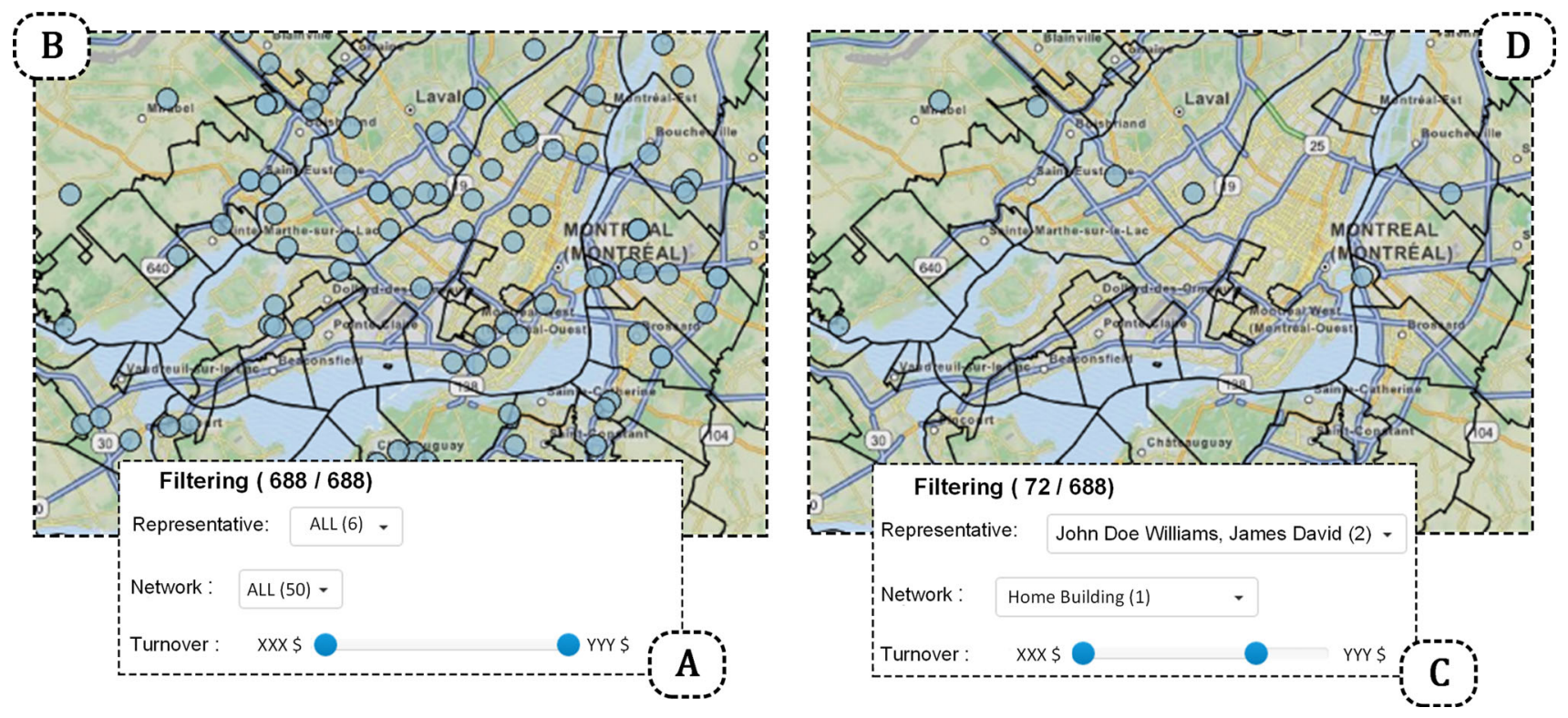

Fig. 9 Filtering options

Fig. 10 Polygon simplification
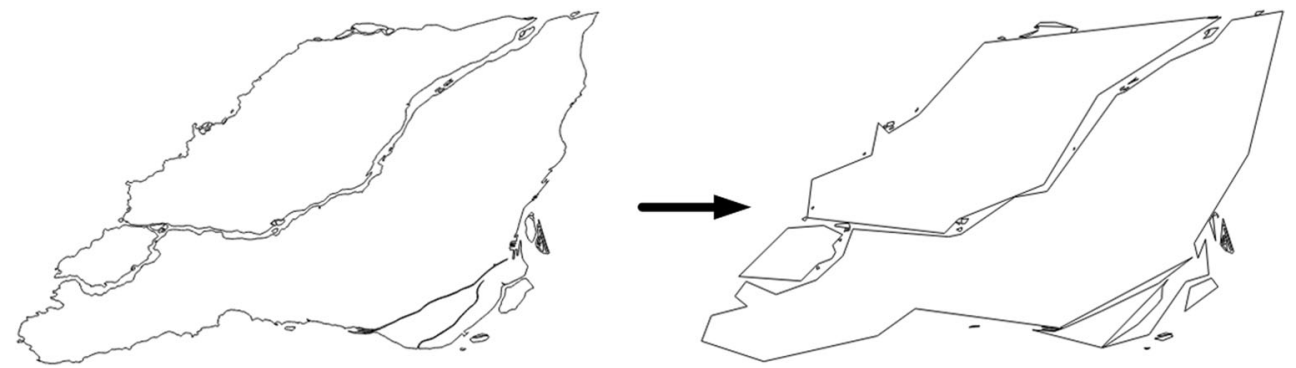

\subsubsection{F5 - Visualization of Different Sales Performance Metrics}

Need Sales assessments are not based on the only metric included at this time (yearly sales); other data is required. Pairwise period sales comparisons or period sales to objective comparisons are metrics that are helpful to correctly assess sales performances.

The need to visualize those metrics through detailed or aggregate format (respectively, retailers and areas) is held and the corresponding capabilities were developed.

Methodology Sales metrics are based on many different periods and computing periodical sales requires a dedicated query. The data has been transformed to a format allowing for temporal queries. The sales data was transformed to have one record for each month per retailer. The data transformation is presented in Fig. 11, allowing a configurable temporal query to be processed, such as in Fig. 12. This transformation also allows the sales data to be easily aggregated at different scales (such as quarterly or annually), thus making it possible to relate it to other temporal data (such as the construction data).

For this, the development phase essentially consisted of developing the configuration interface and the association of each metric with a colorimetric scale. Indeed, as it is not practical to define a colorimetric scale for each metric, a dedicated tool that automatically computes color gradients from the data was developed and integrated in our web interfaces toolbox.

Results Available sales metrics for areas and retailers are now as follows:

- Period sales: monthly, quarterly, yearly, or at year to date.

- Period sales comparison: monthly, quarterly, yearly, or at year to date.

- To objective comparison: monthly, quarterly, yearly, or at year to date.

The displayed metrics are directly configurable from the user interface. 


\begin{tabular}{|c|c|c|c|c|c|}
\hline name & address & sales jan-13 & sales feb - 13 & $\ldots$ & sales dec - 14 \\
\hline J.D Materials & $\ldots$ & $150 \$$ & $220 \$$ & $\ldots$ & $330 \$$ \\
\hline
\end{tabular}

\begin{tabular}{|c|c|c|c|}
\hline name & address & sales month & sales amount \\
\hline J.D Materials & $\ldots$ & janv-13 & $150 \$$ \\
\hline J.D Materials & $\ldots$ & feb-13 & $220 \$$ \\
\hline$\ldots$ & $\ldots$ & $\ldots$ & $\ldots$ \\
\hline J.D Materials & $\ldots$ & dec-14 & $330 \$$ \\
\hline
\end{tabular}

Fig. 11 Table transformation to allow temporal queries

\subsubsection{F6 - Improved User Experience by Providing Access to Contextual Information}

Need Still with the aim of improving spatial understanding of sales performances, the next application focuses on interaction and visualization capabilities.

The colorimetric scales provide a first assessment of the metrics studied. The focus now is to have quick access to the evaluated metrics. Moreover, charts have been integrated to provide an enhanced visual evaluation.

Methodology In addition to the OpenLayers (2016) and Bootstrap (2016) libraries, Chart.js (2016) is added for chart management. Interfaces were developed to conform to the input requirements of each library.

To allow for future adaptations, all new interfaces were developed taking into consideration flexibility. For example, retailers' styling is decomposed into multiple steps: retrieve coloring variable, get associated data, define corresponding color scale, and render the retailer's dedicated point. If a change happens in the selected colored variable, the information is automatically propagated to the next steps.

Results Selected metrics are now directly accessible when hovering over the desired retailer (Fig. 13d). A clicking interaction now allows for information that is relevant to the partner's decision makers to be shown.

In Fig. 13, selected areas are now highlighted Fig. (13a), and the current observed metric of the selected area is also presented at the top (Fig. 13c) of the information panel (Fig. 13b). As one important assessment metric is a comparison to the objectives, a bar chart shows sales of the past and current years in parallel with fixed objectives.

\subsubsection{F7 - Investigation of Environmental Data}

Need Local environment is an important factor in the performance of the retail industry. Some areas are more favorable than others, depending on local socio-demographic factors or other parameters.

According to our partner, the three main fields where we must focus our attention were construction data, socio demographic data, and certain points of interest, such as competitors, ski resorts and golf clubs.

While a future objective is to process KDD (Knowledge Discovery from Databases) methods on those datasets, the partner was actually interested in having the ability to to access to that information through a dedicated application.

Furthermore, those datasets contain various data that are not all useful for each case study, so it is pertinent to determine what information is relevant. In this case, median income (Census Data) and construction building dates (National Household Survey (NHS) data) were some of the selected data collected in the application.

Methodology The data acquisition on socio-demographic information was done from two main sources, the Census Data (Statistics-Canada 2016) and the NHS (Statistics-Canada 2016). Those two datasets can be found in the adapted aggregation level (CSD for this application), so only a basic data treatment had to be processed before integration. For these two datasets, the data retrieved was the most recent available at the time of the study, those of the 2011 census.

Construction data was acquired from the Census Database and the Canadian Mortgage and Household Corporation CMHC (2016). Those datasets had to be cleaned and transformed before being integrated in the database.

For the different points of interest, such as competitors, ski resorts and golf clubs, no available dataset was found. Nevertheless, that information is available through the web: competitors' websites often offer store location webpages, and ski resorts and golf clubs are listed along with their address on dedicated websites.

As the need to acquire data from websites appeared, tools to process data acquisition form websites were gathered and integrated in our toolkit. For example, http queries library (Java.net.URL) to automatize webpage access and source downloads (in html or other format). HTML parsing packages were also used (Jsoup 2016). Finally, when needed, addresses were converted to
Fig. 12 Configurable temporal query

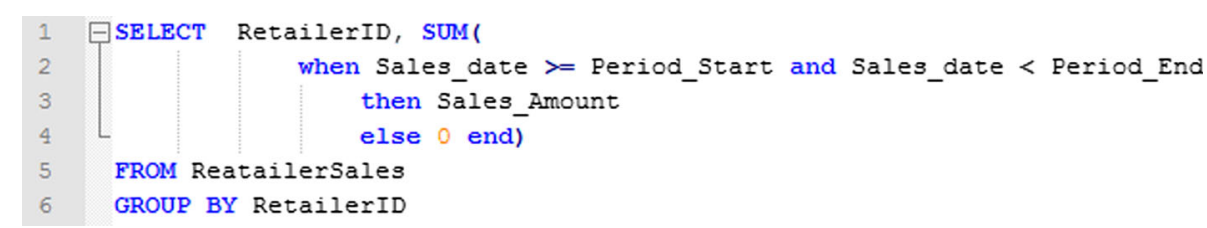




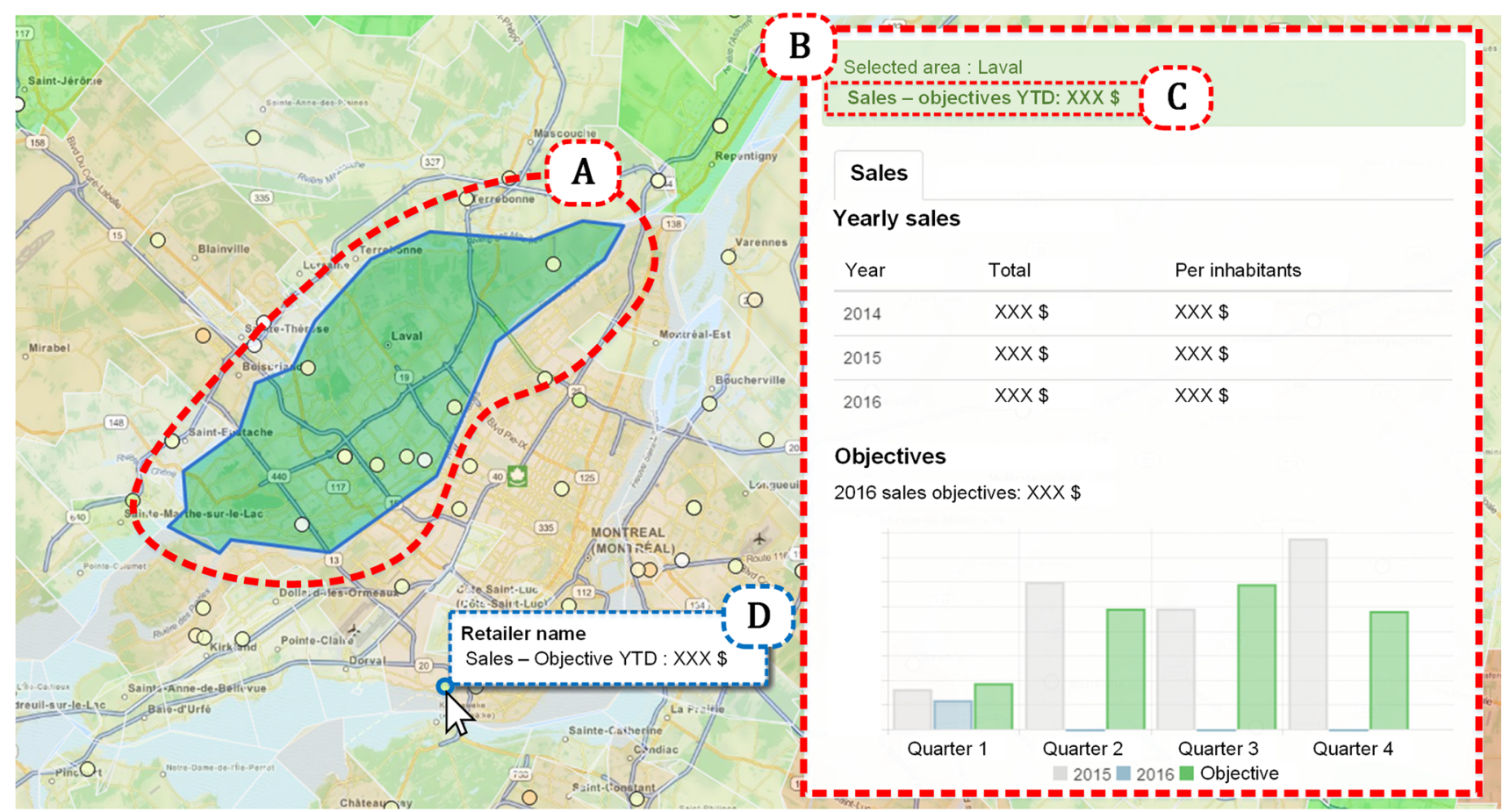

Fig. 13 User interface capabilities

geographical coordinates using the tools developed previously (in F1). Thus, it was possible to gather the needed data and to group it into dedicated dataset layers. Here, the main development consists of the integration of the access to the data through adapted interfaces.

Results Previously this data was accessible in unsuitable formats, and through a separate platform, and mixed with other irrelevant data. Now the current interface allows for the relevant sources of data to be investigated:

- number and amount of building permits (monthly per CSD),

- housing starts number by type (quarterly per CSD),

- Census Data (detailed per CSD),

- National Household Survey Data (detailed per CSD),

- ski resorts, golf club and competitor locations.

Exploring those datasets can now be done more easily by accessing the detailed information in each area (details are accessible by clicking on the desired area). It is also possible to show dataset elements on the map by selecting the dedicated layer: competitors, ski resorts and golf clubs.

\subsection{Extension to Other Datasets}

This section shows how the developed SDSS is easily adaptable to other datasets. Here, a complete transfer to the Province of Ontario is exemplified. For this transfer, the partner provided sale and retailer data for the Province of Ontario. It must be noted that the new datasets gathered for the province of Ontario have the same structures as those used for the province of Quebec. According to the proposed method (step A) the input data has been transformed, cleaned and processed with the tools already developed (address geocoding (in Fl), table transformations (in $F 5$ ), etc.). Then, that data was integrated into the database. Step C: Ontario CSD file limits were downloaded, and a simplified version was computed with QGIS and integrated into the database (as in F4). Data queries for sales aggregation (in $F 2$ ) or periodical queries (in $F 2$ and $F 5$ ) were developed generically. Then, everything that was needed to provide data to the user interface was already available. The interface between the new data and the flexible interface from $F 6$ was configured.

While setting up the first platform for the province of Quebec took about four months for a single engineer, setting up the platform for the Province of Ontario, taking advantage of the previously-developed tools, took twelve working hours (also for one engineer). The new platform allows our partner to visualize and investigate sales in Ontario through the same type of interface (Fig. 14).

\section{Conclusion}

As can be understood from the literature review, and particularly in Table 1, SDSS may be useful for many applications. Moreover, there is a constantly growing volume of 


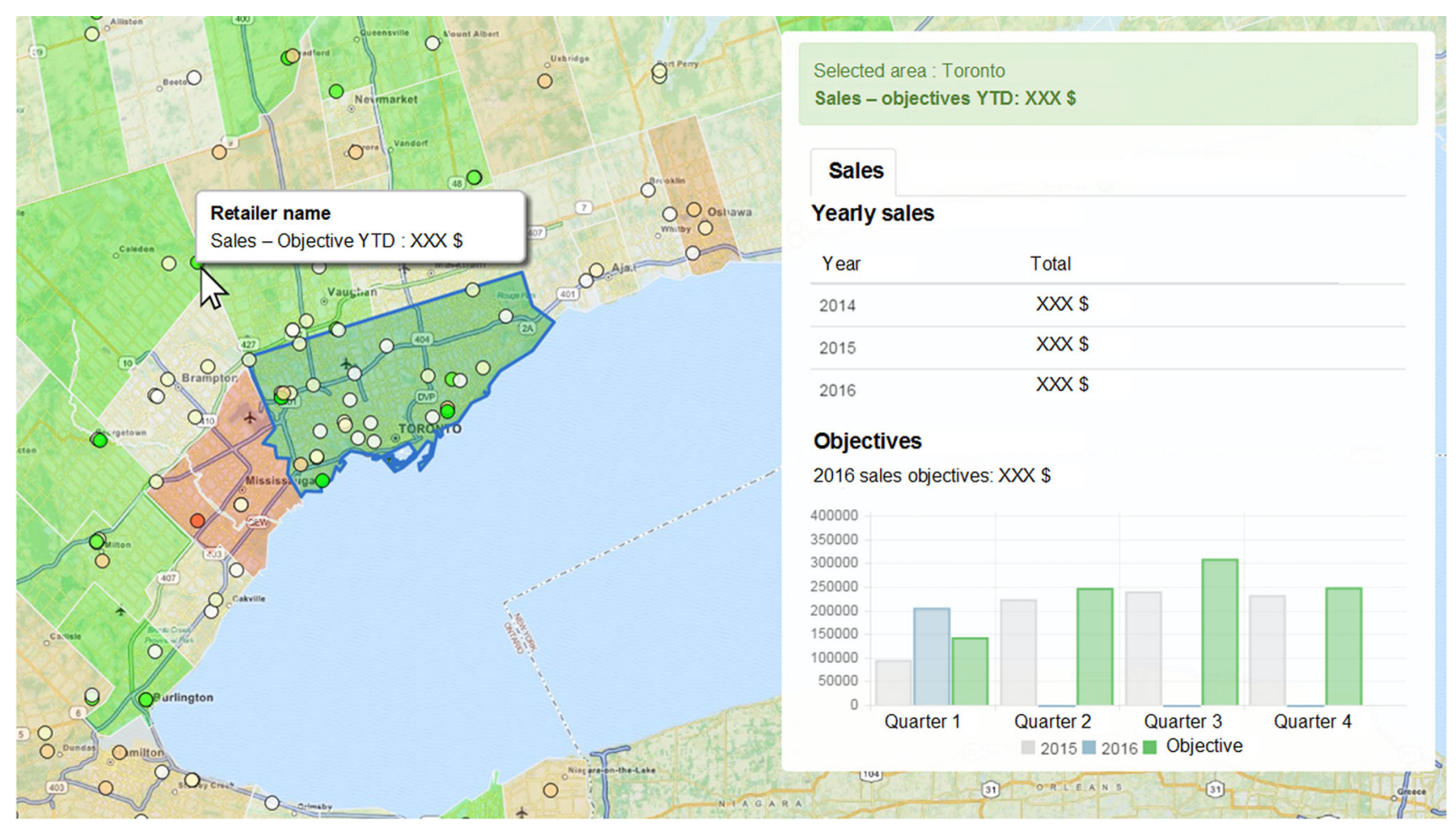

Fig. 14 Transfer to the Province of Ontario

geospatial data stored in various public or private domains that makes new areas of development possible.

Furthermore, the current state of the art only offers partial solutions to these needs: on the one hand, generic methodologies are available and on the other hand there are case-based specific applications but no reproducible solution.

The proposed approach allows for the development of many SDSS in an integrated process framework and technology architecture. This proposal relies on a conceptual framework composed of a SDSS architecture and a development approach. The architecture relies on two categories of clients (Thick clients and Thin clients). Tools are proposed for the Thick clients so they may easily manipulate the data in the SDSS for any analysis and/or develop applications for the Thin clients. Moreover, the proposed development approach makes it possible to factor in different tasks of the development in a case where several dedicated applications have to be developed for the same business

To summarize, the proposed framework offers:

- an integrated solution for the manipulation of geospatial data,

- a centralized system to integrate various sources of data,

- an open solution, independent of any proprietary system,
- a flexible solution that adapts to many case studies, and

- a solution that is considered very efficient for the case study (feedback from our industrial partner).

The framework has been validated on a real case study, and we presented a detailed explanation to show the development of a specific functional SDSS.

Benefits for scholars include the availability of a integrated framework that permit, at the same time, for each one, to manipulate, develop and test, in parallel, multiple decision tools, directly on the dataset. It is easy to include new tools and validate their usefulness with the partner. For the practitioners, it provides a set of tools that offers an integrated view of the data that is automatically prepared and presented graphically.

Future developments will focus mainly on the development of various tools that allow for the analysis of data in various case studies. Data mining and Operations Research techniques will be adapted and integrated within the SDSS. Evaluation methods to assess local and global performances adapted to the case study will be developed; for example, metrics to evaluate the sales potential among the territory. This will lead to maximizing demand coverage under constraints that are specific to this application.

The proposed approach has limitations. The proposed architecture does not take into account some constraints that can appear in cases of academic or industrial development: - scalability, the proposed architectural approach 
does not take into account resource constraints when used on a large scale (number of users, amount of data, etc.) continuous data flow: the proposed approach is not yet adapted to the continuous data integration and analysis; consideration of this aspect would be relevant in future research. - Aspects related to data security are not addressed, some scientific or industrial sectors may have to ensure the confidentiality and integrity of the data. - There may be constraints on the use of proprietary software for carrying out the analyses, the interfaces with the proposed system would have to be put in place.

The SDSS developed and presented in the case study is currently in use by our industrial partner, and the results from our future analysis will be plugged into the current platform.

Acknowledgements The authors acknowledge the support offered by the research consortium FORAC (Forest to Customer) (2016) and Maibec (2016) that, in addition to all of the data used in the case study, provided comments and answers to many questions during the project.

\section{References}

Adejuwon A, Mosavi A (2010) Domain driven data mining: application to business. Int J Comput Sci 7(4):41-44

Agrawal S, Gupta RD (2014) Development and comparison of open source based web GIS frameworks on WAMP and apache tomcat web servers. In: ISPRS -international archives of the photogrammetry, remote sensing and spatial information sciences XL-4, pp 1-5

Armstrong M, De S, Densham PJ, Lolonis P, Rushton G, Tewari V (1990) A knowledge-based approach for supporting locational decisionmaking. Environ Plan B Plan Design 17(3):341-364

Benoit D, Clarke GP (1997) Assessing GIS for retail location planning. J Retail Consum Serv 4(4):239-258

Bogorny V, Martins Engel P, Alvares LO (2005) A reuse-based spatial data preparation framework for data mining. In: Proceedings of the 17th international conference on software engineering and knowledge engineering (SEKE'2005), pp 649-652

Bootstrap. http://getbootstrap.com/. Accessed 13 July 2016

BoundlessGeo. http://boundlessgeo.com/. Accessed 13 July 2016

Bradlow ET, Gangwar M, Kopalle P, Voleti S (2017) The role of big data and predictive analytics in retailing. J Retail 93(1):79-95

Chart JS. http://www.chartjs.org/. Accessed 13 July 2016

Chen J, Maceachren AM, Guo D (2008) Supporting the process of exploring and interpreting space-time multivariate patterns: the visual inquiry toolkit. Cartogr Geogr Inf Sci 35(1):33-50

Cios K, Pedrycz W, Swiniarski R, Kurgan L (2007) The knowledge discovery process. In: Data mining: a knowledge discovery approach. Springer Science \& Business Media, Berlin, pp 9-24

Cliquet G, Fady A, Basset G (2006) Management de la distribution, 2nd edn. Dunod, Paris

CMHC. https://www.cmhc-schl.gc.ca/. Accessed 13 July 2016

Crossland M, Wynne B, Perkins W (1995) Spatial decision support systems: an overview of technology and a test of efficacy. Decis Support Syst 14(3):219-235
Densham P (1991) Spatial decisions support systems. In: Longley P (ed) Geographical information systems: principles and applications. Wiley, Hoboken, pp 403-412

Dubelaar C, Bhargava M, Ferrarin D (2002) Measuring retail productivity: What really matters? J Bus Res 55(5):417-426

Erskine M, Gregg D, Karimi J, Scott J (2013) Business decisionmaking using geospatial data: a research framework and literature review. Axioms 3(1):10-30

Evans B, Sabel CE (2012) Open-source web-based geographical information system for health exposure assessment. Int J Health Geogr 11:1-11

Fayyad U, Piatetsky-Shapiro G, Smyth P (1996) From data mining to knowledge discovery in databases. AI Mag 17(3):37-54

Flowerdrew R (1991) Spatial data integration. Geogr Inf Syst $1: 375-387$

FORAC. http://www.forac.ulaval.ca/. Accessed 13 July 2016

GeoExplorer. http://suite.opengeo.org/opengeo-docs/geoexplorer/. Accessed 13 July 2016

GeoServer. http://geoserver.org/. Accessed 13 July 2016

Ghaemi P, Swift J, Sister C, Wilson JP, Wolch J (2009) Design and implementation of a web-based platform to support interactive environmental planning. Comput Environ Urban Syst 33(6):482-491

Golf-clubs. http://www.legolfquebecois.com/. Accessed 13 July 2016

Google. Google maps api. https://developers.google.com/maps/. Accessed 13 July 2016

Google. Google maps geocoding api. https://developers.google.com/ maps/documentation/geocoding/start. Accessed 13 July 2016

Granell C, Díaz L, Gould M (2010) Service-oriented applications for environmental models: reusable geospatial services. Environ Model Softw 25(2):182-198

Hernandez T (2007) Enhancing retail location decision support: the development and application of geovisualization. J Retail Consum Serv 14(4):249-258

Hess R, Rubin R, West L (2004) Geographic information systems as a marketing information system technology. Decis Support Syst 38(2):197-212

Jin X (2011) A supply chain optimization DSS web-services-based for e-retail industry. In: 2011 IEEE power engineering and automation conference (PEAM). IEEE, Wuhan, pp 229-232

Jsoup. https://jsoup.org/. Accessed 13 July 2016

Keenan P (2004) Using a GIS as a DSS generator. ICFAI University Press, Hyderabad, pp 97-113

Keenan P (2006) Spatial decision support systems: a coming of age. Control Cybern 35:9-27

Khan M, Khan SS (2011) Data and information visualization methods, and interactive mechanisms: a survey. Int J Comput Appl 34:1-14

Knezic S, Mladineo N (2006) GIS-based dss for priority setting in humanitarian mine-action. Int J Geogr Inf Sci 20(5):565-588

Lloyd R (1997) Spatial cognition: geographic environments, vol 39. The GeoJournal library. Springer, Dordrecht

Loucks DP (1995) Developing and implementing decision support systems: a critique and a challenge. J Am Water Resour Assoc 31(4):571-582

MacEachren AM, Kraak M-J (2001) Research challenges in geovisualization. Cartogr Geogr Inf Sci 28(1):3-12

Maibec. http://www.maibec.com/. Accessed 13 July 2016

Mendes A, Themido I (2004) Multi-outlet retail site location assessment. Int Trans Oper Res 11(1):1-18

Mennis J, Guo D (2009) Spatial data mining and geographic knowledge discovery-an introduction. Comput Environ Urban Syst 33(6):403-408

Moreno-Sanchez R, Anderson G, Cruz J, Hayden M (2007) The potential for the use of open source software and open 
specifications in creating web-based cross-border health spatial information systems. Int J Geogr Inf Sci 21(10):1135-1163

OpenLayers. http://openlayers.org/. Accessed 13 July 2016

Otto B (2011) Data governance. Bus Inf Syst Eng 3(4):241-244

PgAdmin. http://www.pgadmin.org/. Accessed 13 July 2016

PostGIS. http://postgis.net/. Accessed 13 July 2016

PostgreSQL. https://www.postgresql.org/. Accessed 13 July 2016

QGIS. http://www.qgis.org/en/site/. Accessed 13 July 2016

R. http://www.r-project.org/. Accessed 13 July 2016

Reinartz W, Dellaert B, Krafft M, Kumar V, Varadarajan R (2011) Retailing innovations in a globalizing retail market environment. J Retail 87:S53-S66

Rey S (2009) Show me the code: spatial analysis and open source. J Geogr Syst 11(2):191-207

Rinner C, Keßler C, Andrulis S (2008) The use of web 2.0 concepts to support deliberation in spatial decision-making. Comput Environ Urban Syst 32(5):386-395

Roig-Tierno N, Baviera-Puig A, Buitrago-Vera J, Mas-Verdu F (2013) The retail site location decision process using gis and the analytical hierarchy process. Appl Geogr 40:191-198

Sikder IU (2009) Knowledge-based spatial decision support systems: an assessment of environmental adaptability of crops. Expert Syst Appl 36(3):5341-5347

Ski-resort. http://www.maneige.com/. Accessed 13 July 2016

Sprague RH (1980) A framework for the development of decision support systems. MIS Q 4(4):1-26
Statistics-Canada. https://www12.statcan.gc.ca/. Accessed 13 July 2016

Studio R. https://www.rstudio.com/. Accessed 24 October 2016

Sugumaran V (2007) Web-based spatial decision support systems (websdss): evolution, architecture, examples and challenges. Commun Assoc Inf Syst 19:844-875

Thompson A, Walker J (2005) Retail network planning: achieving competitive advantage through geographical analysis. J Target Meas Anal Mark 13(3):250-257

Tomcat. http://tomcat.apache.org/. Accessed 13 July 2016

Vatsavai RR, Shekhar S, Burk TE, Lime S (2006) Umn-mapserver: a high-performance, interoperable, and open source web mapping and geo-spatial analysis system. In: Raubal M, Miller HJ, Frank AU, Goodchild MF (eds) Geographic information science: 4th international conference, GIScience 2006, Munster, Germany, 20-23 Sept, vol 4197. Springer, Berlin, pp 400-417

Wanderer T, Herle S (2015) Creating a spatial multi-criteria decision support system for energy related integrated environmental impact assessment. Environ Impact Assess Rev 52:2-8

Yeoman. http://yeoman.io/. Accessed 13 July 2016

Zhang C, Zhao T, Li W (2010) The framework of a geospatial semantic web-based spatial decision support system for digital earth. Int J Digit Earth 3(2):111-134

Zhu X, Healey R, Aspinall R (1998) A knowledge-based systems approach to design of spatial decision support systems for environmental management. Environ Manag 22(1):35-48 